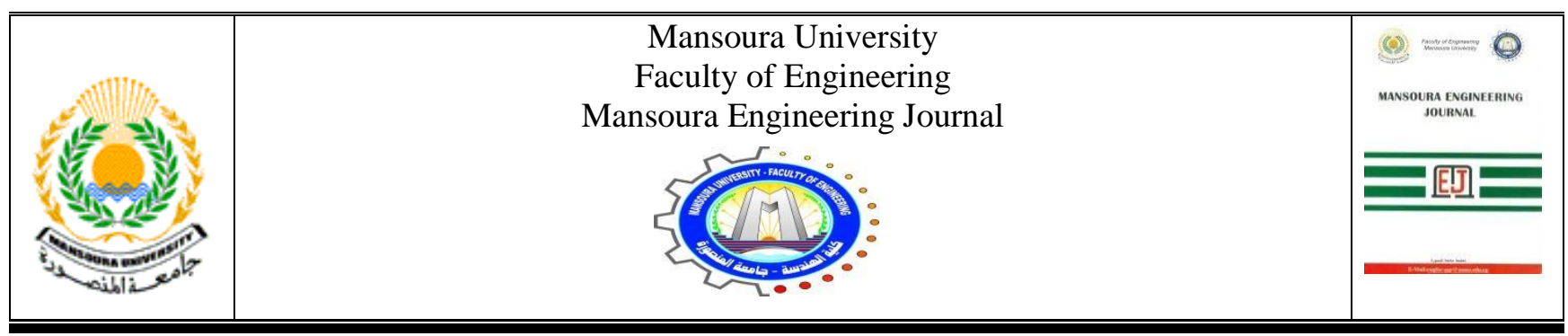

\title{
Online Quality Control System in Single Jersey Circular Knitting Machine
}

\author{
A. Fouda
}

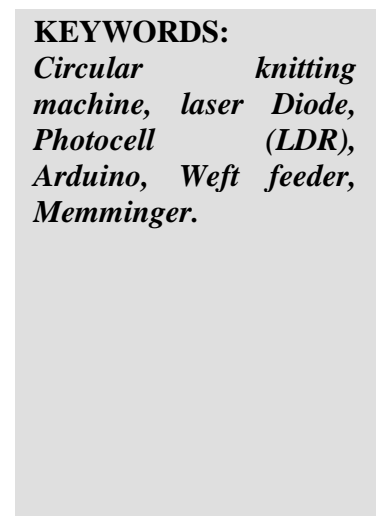

\begin{abstract}
With the great development in the technology of manufacturing knitting machines, and high productivity. There are still some drawbacks that might be overcome by applying novel measurement methods, particularly, in the circular single Jersey knitting machines. The most important of these drawbacks is the laddering defects. In this paper, an online quality control system - the first of its kind - was developed and assembled on the machine and consists of a transmission unit (laser) and a receiving unit (photocell), to detect laddering defects in real-time. Once occurred, the system beeps to draw the attention of the operator and the machine stops automatically, the machine then runs again after the defect is being fixed. Thus, this system can reduce the production of the second degree, raise the level of quality, and reduce the production costs per kilograms of the produced fabric. This system proved its sensitivity and efficiency while the machine runs at the typical production speeds.
\end{abstract}

\section{INTRODUCTION}

Recently, the unconventional uses of knitted fabrics have multiplied, due to the large productivity and recent developments in knitting machines, particularly, the circular knitting machines. However, dealing with defects arising while production necessitates advanced studies and research, in line with the technological development in measurement methods and techniques.

It is economical to produce high quality knitted fabrics, this necessitates introducing more advanced technology in the knitting industry. The customer is expected to seek better quality at a reasonable price [1]. Consequently, kitted fabric producers need to keep up their place in the garments market by improving the quality as well as maximizing productivity. Nevertheless, running the knitting machine at a higher speed

Submit: 6 August 2020 - Revised: 26 August 2020 - Accepted: 30 August 2020

Abd Elmonem Fouda, Textile Engineering Department, Faculty of Engineering, Mansoura University, Mansoura, 35516, Egypt

E-mail:eabdo3@gmail.com may create many fabric defects that would be used as a way to bargain the fabric price. Hence, novel inspection techniques shall be utilized to guarantee the knitted fabric quality, and consequently, the on-time defect-recognition system turns out to be a higher priority than any other time [1].

Usually, a good quality control system intends to make a significant contribution to saving both time and money. To correct the process faults, when occurs, the circular knitting machine must be stopped, the production cost decreases. This, of course, raises the manufacturing cost and may also increase the cost of the fabric finishing process, thus, the overall manufacturing cost increases. Moreover, if quality requirements are not met, the knitted fabric may be rejected, which would have a negative impact on the reputation of the mill [1]. Generally, the knitted fabric defects can be classified into vertical and horizontal faults. This defects typically results from imperfections in yarns, incorrect knitting settings, poor machine conditions, lack of maintenance, inadequate monitoring of knitting machine performance, or inappropriate yarn delivery $[1,2,3]$.

To minimize the knitting defects, producers attempted to 
apply quality control by using different detection devices. One of the models incorporated a needle finder to identify the butt brooked needles, another system successfully identified any broken yarn during the knitting process by using a yarn breaking sensor. Such devices contribute to improving fabric quality. Nevertheless, better techniques are utilized to screen the key knitting parameters and interfere quickly to apply preset steps when deviations are identified $[1,2,3]$.

The online quality systems depend mainly on measuring the most significant knitting performance parameters continuously throughout the knitting process and comparing these data to a predefined set of information that realizes a minimum targeted quality. Theses parameters include production process variables including but not limited to yarn input tension, yarn irregularity, yarn delivery speed, etc. [4].

Furthermore, other online quality systems depend on fabric image analysis. Samples of faulty knitted raw fabrics were captured by an advanced camera. The captured images are then converted into a grey level image via an analog-to-digital converter. Those pictures were analyzed by an image processing technique that includes algorithms and filters to identify the fabric faults. For instance, a drop-stitch fault (a hole), may be recognized by applying a type of filters that efficiently averages out any region in the picture of different light intensity. This means that the little light comes through the little pores is discarded, however, the areas that passed the greater amount of light as a result of a fabric hole were obvious and easily identifiable, and consequently, the fabric defect was successfully recognized $[5,6,7,8]$.

Hemdan A., et al. presented a fabric inspection system valid for circular knitting machine that is upgradeable and can be produced at a reasonable cost. That inspection system differs from others, it was dedicated to a circular knitting machine, and furthermore, it was equipped with a new imperfection recognition method, which has been verified under practical conditions. The proposed system demonstrated a high recognition rate, accuracy, and a low pace of bogus defect alerts [4]. However, that technique was applied to a system that simulated a knitting machine, rather than a real knitting machine.

The laddering defects are caused by broken needles (hook, latch, or butt) also, show noticeably as parallel vertical lines to the fabric wales. When there is a broken needle hook, no loops are formed. And it is the most frequent and visible defect in the machine and causes many of the fabrics produced to be second quality, and consequently, the production cost rises.

Based on the above-mentioned literature review, and due to the shortage of research on online quality control systems used on circular knitting machines, this research presents an attempt to establish an online inspection system that focuses mainly on the laddering defect. Since identifying this defect during production and to stop the circular single Jersey knitting machine once it occurs would improve the quality and saves the cost significantly, by reducing the amount of defected knitted fabrics.

\section{SYSTEM DEVELOPMENT}

To construct the online system to detect the fabric laddering shown in Figure 1, single Jersey knitted fabrics were produced by using a $32 \mathrm{Ne}$ Cotton yarn and loop length of 2.8 mm. ALBI circular single Jersey knitting machine was used (gauge 28, diameter 17 inches, number of feeders 34 and 30 r.p.m).

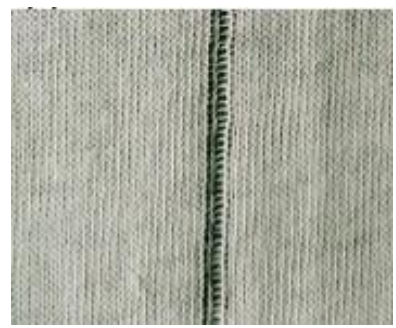

Fig. 1: Laddering defect in knitted fabrics

Figure 2 shows a schematic diagram of the used circular knitting machine, where only major machine parts are shown.

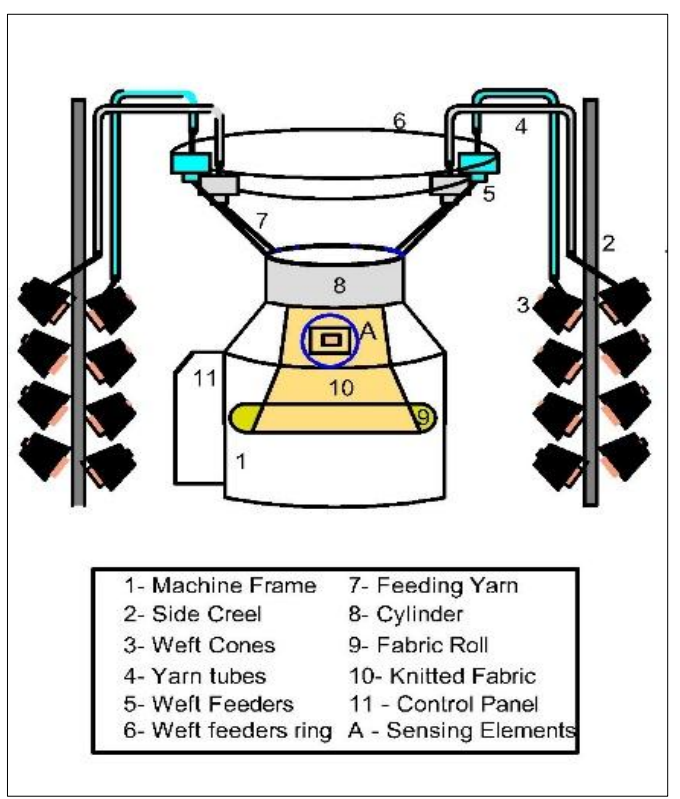

Fig. 2: Diagrammatic sketch of the circular knitting machine used

To identify the laddering defect during the production process, i.e., while the machine is running, the sensing elements (A), shown in Figure 2 has been installed and enlarged in Figure 3 to show its components which consists of a laser pen (2) and its specifications is $(5 \mathrm{~mW} 650 \mathrm{~nm}$ Red Line Laser Diode). The laser pen emits the laser beam (3), which in turn, reaches the receiving unit (5), consists of an LDR (Light Dependent Sensor) photocell and its specifications are as follows; maximum power: $200 \mathrm{~mW}$, maximum voltage: $150 \mathrm{~V}$, light resistance: $\sim 1 \mathrm{k}$ Ohm and dark resistance: $\sim 10 \mathrm{k} \mathrm{Ohm}$. 


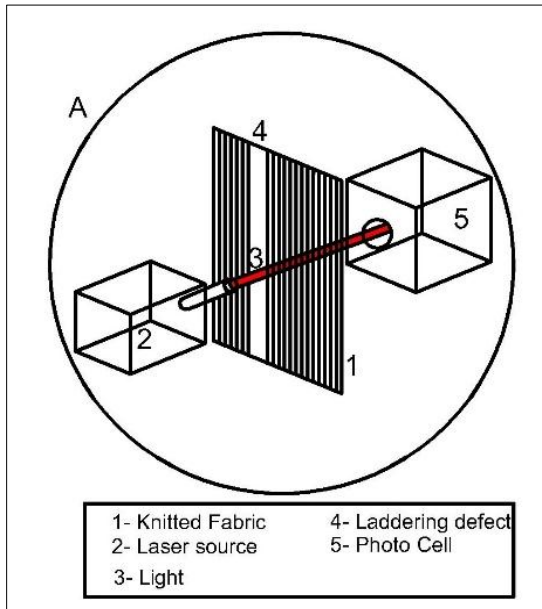

Fig. 3: Magnified diagrammatic sketch of the inspection system, A.

Both laser pen and photocell are connected to the Arduino Uno board, a microcontroller board based on the ATmega328P shown in Figure 4. It has the following specifications; USB connection, a power jack, a reset button. 6 analog inputs, 14 digital input/output pins, of which 6 can be used as PWM outputs, an ICSP header and $16 \mathrm{MHz}$ quartz crystal.

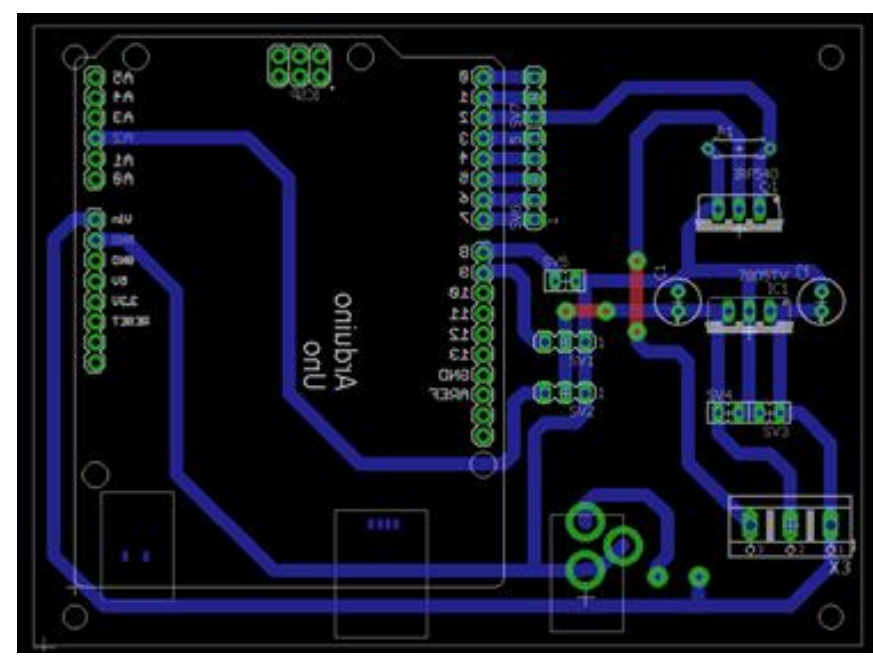

Fig. 4: Interface circuit with Arduino Uno

The Arduino Uno was programmed by using the IDE Arduino Software. The ATmega328 in the Arduino Uno was programmed by using a bootloader which easily permits transferring new code without the need of a programmer or software specialist and it communicates by using the STK500 convention.

A special carrier for both laser pen and photocell was designed using Solidworks software and manufactured accordingly as shown in Figure 5. That carrier ensures that each of them is properly mounted, as well as facilitates controlling their position accurately throughout the measuring process while the machine is running.

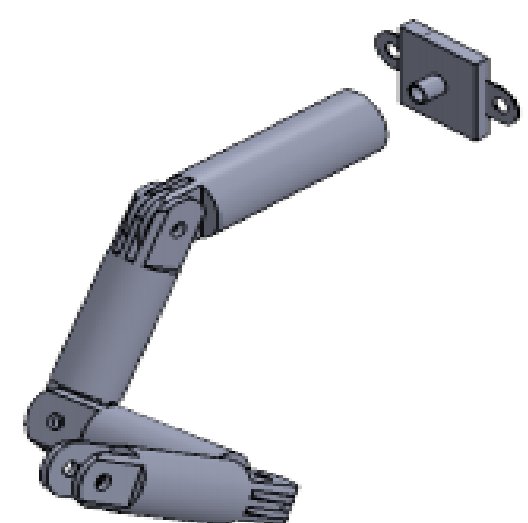

Fig. 5: Carrier of laser source and receiving chamber

\section{MEASURING PRINCIPLE}

The measurement idea is adopted as shown in Figure 3, where the laser pen beam constantly passes through stitches and wales of fabric (1). If the fabric is free of laddering defect, the amount of passing light would be low, which is interpreted by the Arduino circle into a low readout since it has been programmed and a section of its code is shown in Table 1.

TABLE (1)

A SECTION OF ARDUINO UNO CODE

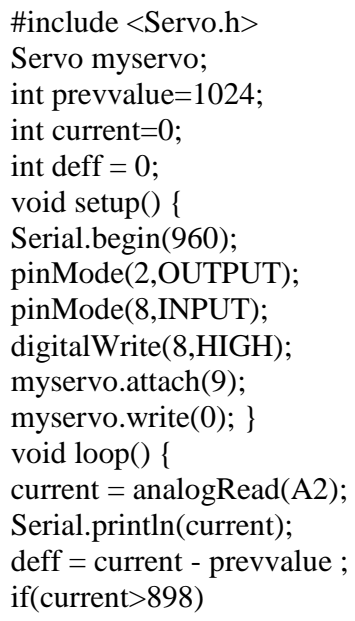

By following the rotation of the knitted fabric tube and in the case of the laddering defect (4), shown in Figure 3, the laser beam (3) passes through the fabric at a greater amount or at a higher concentration towards the photocell which is placed inside a dark chamber to avoid the dispersion of light. Therefore, the value of the readout from the Arduino increases. The Arduino Uno was programmed so that when the Arduino reading value increases, an alarm that has been installed on one of the outputs of the Arduino is triggered and beeps, and the machine stops automatically.

To stop the machine, many procedures have to be carried out. Firstly, the weft feeders or weft memmingers unit shown in Figure 6 was used to make use of its sensing circuit, which in turn works to stop the machine when one of the yarns breaks. An additional weft memminger (K), was installed as 
shown in Figure 6, hence, the total number of wefts Memminger becomes 35 instead of 34 .

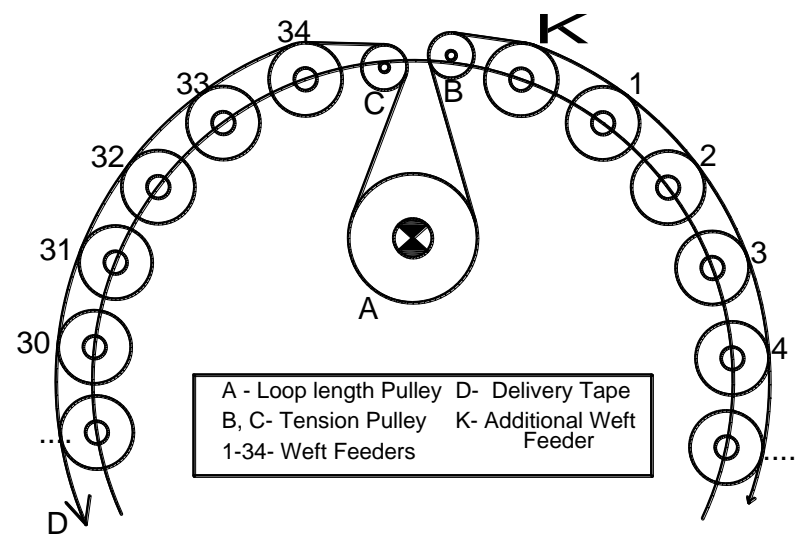

Fig. 6: Feeding unit in the circular knitting machine

Secondly, the servo motor shown in Figure 7 has been used with the specifications given in Table 2. Figure 7 shows the servo motor that is attached to the machine frame (8), it also shows an enlarged view of the additional weft memminger $(\mathrm{K})$, connected to the electrical current tape (1) by the connection points (2). When yarn breakage occurs, either the upper yarn sensor (3) or lower yarn sensor (4) closes the electrical circuit and stops the machine. Since the additional weft memminger $(\mathrm{K})$ is not threaded, so it would always stop the machine and to prevent this, the sensor (3) is disabled, and the sensor (4) has been attached to the servo motor fan (6) by a rope (6).

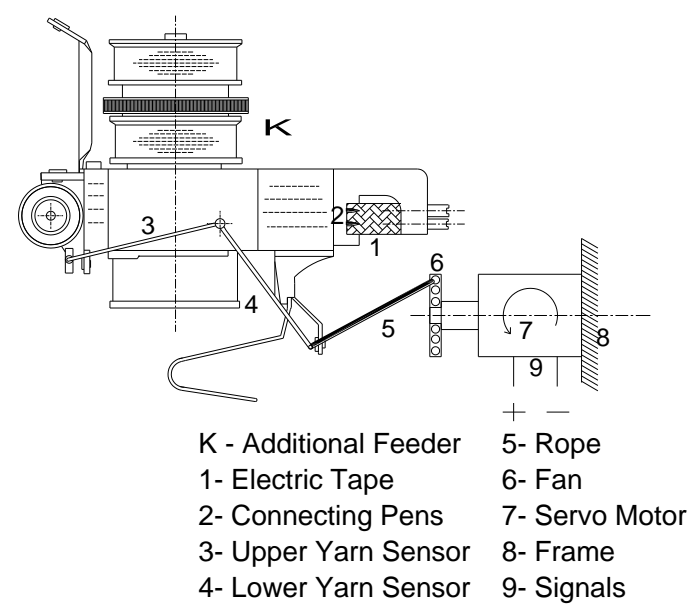

Fig. 7: Weft Memminger and Servo motor

When the defect occurs and the value of the Arduino reading increases, a signal (9) is sent to the servo motor, which rotates and loosens the rope (5), then the sensor (4) shuts off the circuit sensors of the machine, as if a yarn had been broken and the additional feeder lamp is lighted up and the machine stops. The operator notices the defect and replaces the faulty needle, then presses the reset button that was installed in the system as well, which works to send a signal to the servo motor so that it spins back again so that it pulls the rope and the sensor to the top to close the circuit again allowing the machine to run again.
TABLE (2)

SPECIFICATION OF THE SERVO MOTOR USED

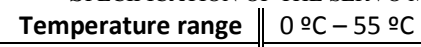

\begin{tabular}{l|l}
\hline Demperature range & \\
\hline Dead bandwidth & $10 \mu \mathrm{s}$ \\
\hline Stall torque & $1.8 \mathrm{kgf.cm}$ \\
\hline Dimension & $22.2 \times 11.8 \times 31 \mathrm{~mm}$, approx. \\
\hline Weight & $9 \mathrm{~g}$ \\
\hline Operating speed & $0.1 \mathrm{~s} / 60$ degree. \\
\cline { 2 - 2 } Operating voltage & $4.8 \mathrm{~V}(\sim 5 \mathrm{~V})$ \\
\hline
\end{tabular}

IV. RESUlts AND DiscuSSIONS

To improve the sensitivity and efficiency of the measuring system, set of problems that affected the quality of the online inspection process, have to be overcome, among which the most important are the following; Firstly, Using an inappropriate laser pen: this problem was overcome by using a laser pen with higher accuracy, to ensure the speed and accuracy of its light passing when the defect occurred. Secondly, Light scattering: It was noted that during the recording process, there is a great difference in Arduino readings, although there is no defect in the fabric. And it turns out that this is caused by the light affecting the photocell. This was overcome by designing a dark chamber for the receiving unit by Solidworks software shown in Figure 5, and it has only an opening for the passage of the laser light through it.

Thirdly, by the rotation of the fabric tube, the distance to the laser pen is observed, and it was noted that the fabric tube takes the form of eye-shape at the sensing point due to the tension of the fabric spreader, which opens the fabric tube after it comes off the cylinder and the needle area, which makes the wales far from each other in a part of the fabric and very close to each other in the knitted fabric tube, this negatively influence the stability of the recording process and affects the quality of the online inspection. The system may give false signals and stop the machine without a reason, also, a defect may pass and the machine doesn't stop. Nevertheless, that was overcome by placing a piece of iron inside the fabric tube and perpendicular to the fabric spreader, to tighten the fabric in the opposite direction, ensuring the uniformity of the wales in the fabric to some extent, consequently, the quality of measurement improved.

During system construction and sensitivity testing, several initial signals were recorded and system sensitivity tested. After developing the measuring system and testing it and removing obstacles that may negatively influence the quality of the measurement and the speed of defect detection, one needle was removed from the machine and the defect was deliberately caused. Figure 8 shows a set of signals that have been recorded. It is noted that the signal rises and falls between a reading range of 4.34 (V) and 4.59 (V) approximately, as a result of the passage of part of the laser light into the optical cell through stitches and wales of nondefective fabric, and this is evident in almost all signals. When the empty needle reaches the online inspection system, the circuit reading increases up to 4.75 (V) approximately, this difference is significant, as the signal is analog, the analog data foe Arduino is $8 \mathrm{Bit}$, so the sensitivity equal to $\frac{5}{2^{8}}=$ $0.019(V)$, but the difference of signals reach to $0.41(\mathrm{~V})$. 

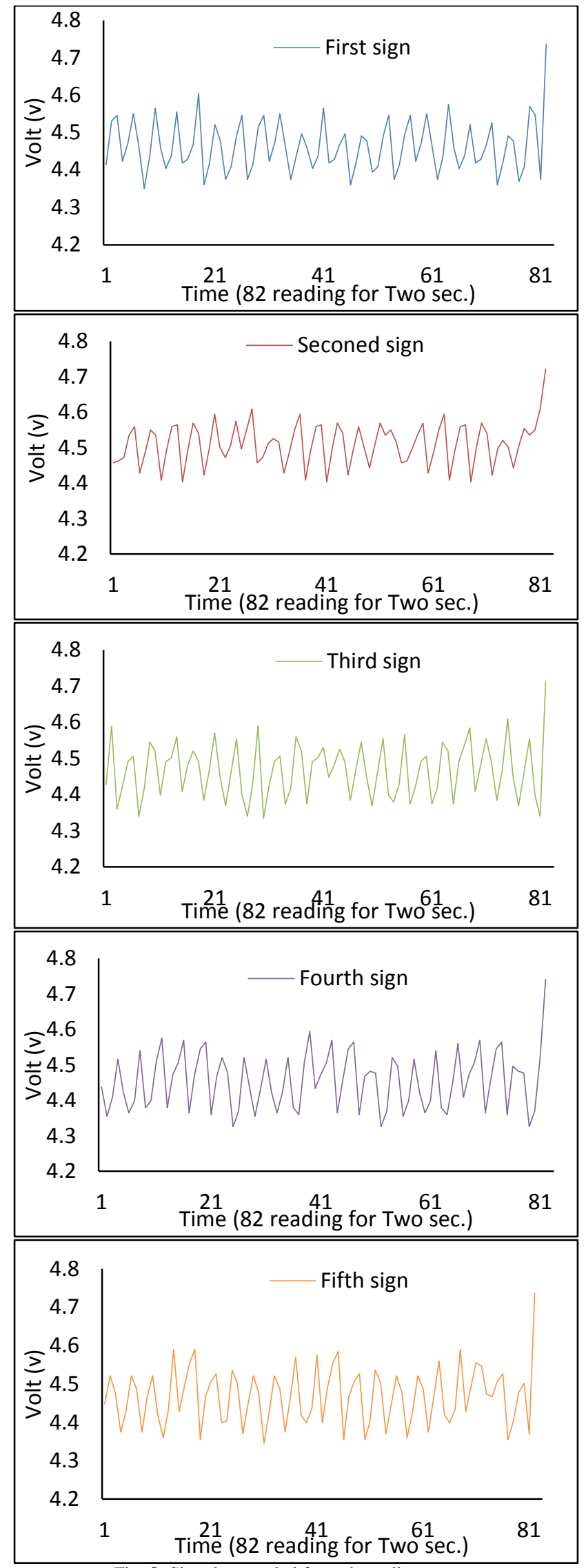

Fig. 8: Signals recorded from the online system
The machine and the recording unit stopped immediately, until the reset button is pressed and the recording process starts again.

\section{CONClusion}

This research presented a practical online system to discover the most common defect of single Jersey circular knitted fabric, which is laddering. By using this system, the percentage of waste and second-degree cloths decreases, where the laddering is discovered immediately upon leaving the machine, and large lengths of fabric with this defect are not produced, consequently, the overall product and process quality increases and the production cost decreases accordingly. It is a unique and first-of-its-kind system that detects a defect online in a real-time compared to other systems that work on models that simulate the knitting machine, or systems that work offline.

\section{REFERENCES:}

1- Catarino A., Araujo M. D. and Hong H." Process control for total quality in circular knitting", AUTEX Research Journal, vol. 1, no. 1, pp. 21-29, 1999.

2- Bailey D. L, "Methods to prevent barré in knitted fabric", Paper presented at the 15th EPS Conference Memphis, TN, June 10-12, 2002.

3- Brackenbury T., "Knitted Clothing Technology", Oxford: Blackwell Scientific Publications, 2002.

4- Hemdan A. and Aya Tallah M., "Online fabric defect detection and full control in a circular knitting machine", AUTEX Research Journal, Vol. 8, No1, pp. 21-29, 2008.

5- Monteiro J., Rocha A. M. and Catarino A. "Monitoring knitting process through yarn input tension: New developments", IECON 28th Annual Conference of the IEEE, vol. 3, pp. 2022-2027, 2002.

6- Monteiro J. L., Catarino A. and Soares F., "Technique for unveiling faults during knitting production", IEEE International Conference on Industrial Technology, 4-7 May, vol. 1, 389-394, 2004.

7- Monteiro J. L., Catarino A., Rocha A. and Soares F., "A system for knitting process monitoring and fault detection on weft circular knitting machines", Paper presented at the World Textile Conference - Fourth AUTEX Conference, Roubaix, June 22-24, 2004.

8- Mcginnity M., Lunney T., Hashim A. and Convery S. "Automated fabric inspection", International Journal of Clothing Science and Technology, vol. 6, no. 5, pp. 15-19, 2002.

\section{Title Arabic:}

$$
\text { منظومة جودة آنية على ماكينات التزيكو الائرية الساده }
$$

\section{Arabic Abstract:}

مع التطور الكبير فى تكنولجيا تصنيع ماكينات التريكو، وزيادة الاتتاجية. لا

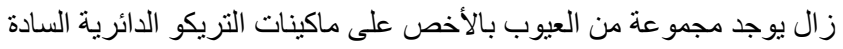

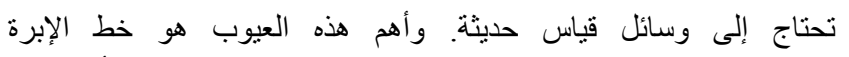

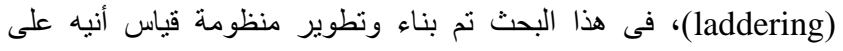

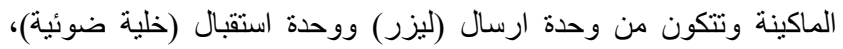

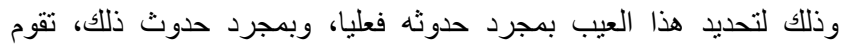

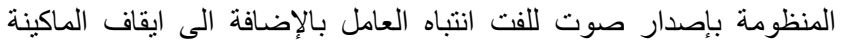

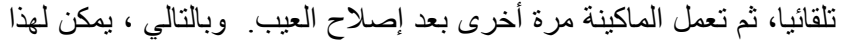

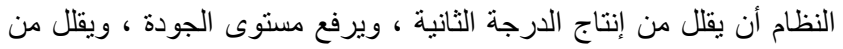

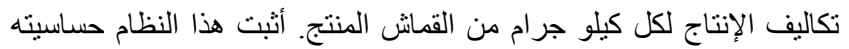
وكفاءته مع السرعات الانتاجية لماكينات التريكو الدائرية الساده. 